\title{
Design and Implementation of Bpsk Modulator and Demodulator Using Vhdl
}

\author{
${ }^{1}$ Hina Malik, ${ }^{2}$ D.R.Rotake, ${ }^{3}$ Mamta Mahajan \\ ${ }^{123}$ Research scholar, G.H.R.I.E.T.W, Nagpur University, M.S,INDIA
}

\begin{abstract}
This paper give the brief description of digital communication system.Digital communication is more reliable, secure and efficient than that of analog communication.In Digital communication,BPSK is most important and efficient technique in terms of signal power. In this paper BPSK modulator and demodulator are purely design by using hardware description language (VHDL) and implementing it on Spartan 3E FPGA kit.
\end{abstract}

\section{Introduction}

In the last years, a major transition from analog to digital modulation techniques has occurred and it can be seen in all areas of wireless communication, satellite and cellular systems.

A digital communication system is more reliable than an analog. The aim of the paper is to create a BPSK (Binary Phase Shift Keying) modulator and demodulator using Xilinx ISE 12.3 software. BPSK consist of modulator a channel and demodulator. The modulated signal was achieved in the first Spartan 3E board, passed through a channel and transmitted to the second board, which behaves as a demodulator.

\section{Theoretical Backgrounds}

\section{A. Digital Communication System:}

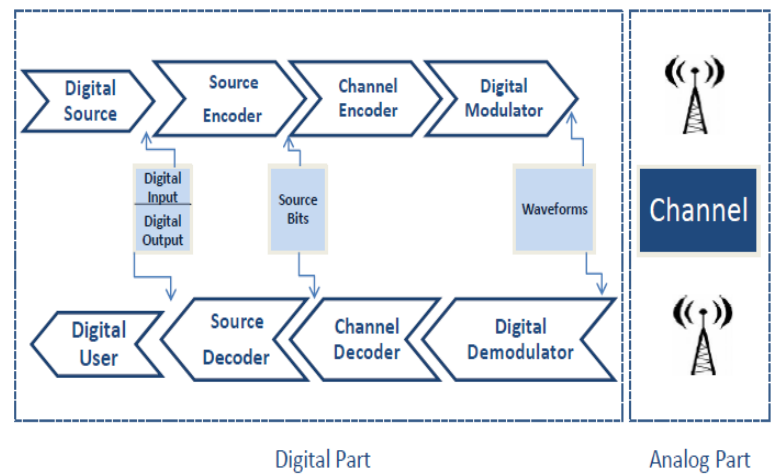

Figure 1. A Digital Communication System

The typical block diagram of digital communication system is presented in Fig.1.The digital communication system consist of both digital and analog parts. The digital part consists of digital source/user, source encoder/ decoder, channel encoder/decoder and the digital modulator or demodulator. The analog part is made of the transmitter, receiver, the channel models and noise models.

The message to be sent is from a digital source, in our case, from a computer. The source encoder accepts the digital data and prepares the source messages. The role of the channel encoder is to map the input symbol sequence into an output symbol sequence. The binary information obtained at the output of the channel encoder is than passed to a digital modulator which serves as interface with the communication channel. The main purpose of the modulator is to translate the discrete symbols into an analog waveform that can be transmitted over the channel. In the receiver, the reverse signal processing happens. A channel is the physical medium that carries a signal between the transmitter and the receiver. The signal is corrupted with noise whatever the medium used for transmission.

In the receiver, the reverse signal processing happens. A channel is the physical medium that carries a signal between the transmitter and the receiver. The signal is corrupted with noise whatever the medium used for transmission. The role of a digital communication system is to transport digital data between the transmitter and receiver. As the signals propagate between the two nodes, they may be submitted to distortion due the channel imperfection. The digital data is transmitted between the transmitter and the receiver by varying a physical characteristic of a sinusoidal carrier, either the frequency or the phase or the amplitude. This operation is performed with a modulator at the transmitting end to impose the physical change to the carrier and a demodulator at the receiving end to detect the resultant modulation on reception. 


\section{B.Basic BPSK Modulator and Demodulator:}

Digital modulation is the process by which digital symbols are transmitted into waveforms that are compatible with the characteristics of the channel. The modulation process converts the signal in order to be compatible with available transmission facilities. At the receiver end, demodulation must be accomplished by recognizing the signals. The modulation technique used in this project is BPSK (Binary Phase Shift Keying) and it is widely used in digital transmission.. BPSK modulation is the simplest form and most robust of all the PSK modulation techniques. The BPSK modulator is quite simple and is illustrated in fig.2. The binary sequence $\mathrm{m}(\mathrm{t})$ or modulating signal is multiplied with a sinusoidal carrier and the BPSK modulated signal $\mathrm{s}(\mathrm{t})$ is obtained. The output of the BPSK signal generated by the modulator is shown in Fig 3.

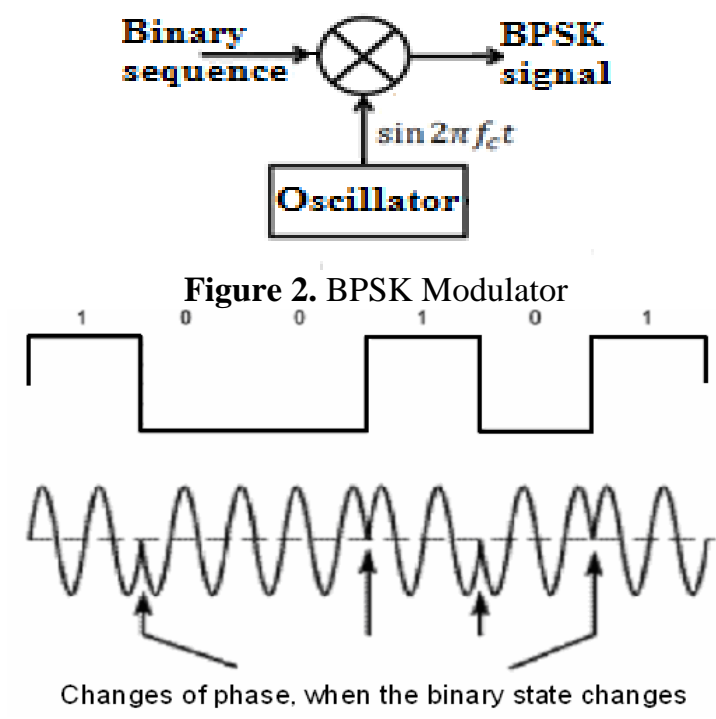

Figure 3. BPSK Modulator output

At the receiver side to demodulate the signal, it is necessary to reconstitute the carrier. This process is made in the Carrier Recovery Circuit. Next, the BPSK modulated signal is multiplied with the carrier, pass through an integrator and then decision circuit will give the modulating signal at the end.

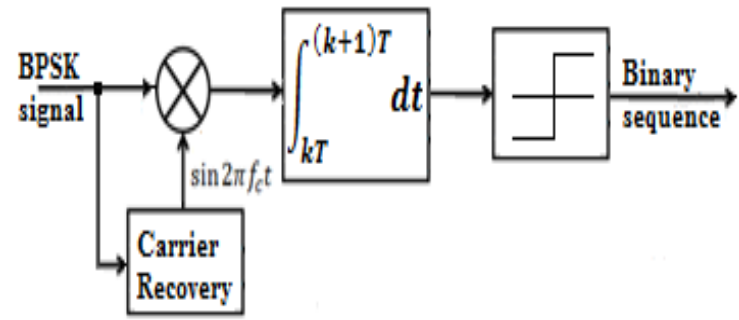

Figure 4. BPSK Demodulator

\section{Design of BPSK Modulator:}

Block diagram of BPSK modulator is shown in figure 5. It consists of DDS Compiler, LFSR, and a mux. The modulating signal which are in the form of binary digits i.e. 0 and 1, are generated internally by a LFSR (Linear Feedback Shift Register). DDS Compiler Block is a direct digital synthesizer which is the main part of the system which is use to generates the carrier i.e sinusoids.DDS uses a lookup table scheme to generate sinusoids. A digital integrator generates a phase that is mapped by the lookup table into the output waveform. The mux block implements a multiplexer. It has one select input and a configurable number of data inputs that can be defined by the user. The $\mathrm{d} 0$ and $\mathrm{d} 1$ inputs of mux represent the sine waves. The sel input of mux represents the modulating signal and selects between the $\mathrm{d} 0$ and $\mathrm{d} 1$ inputs. If LFSR is ' 1 ', the modulated signal remained same as the carrier, but if ' 0 ' was transmitted, then the carrier is shifted by $180^{\circ}$ phase shift. At the output we will get the modulated signal. The expected waveforms at the modulator side are shown in figure 6 . Results of modulator and its different blocks are shown in fig 10. 


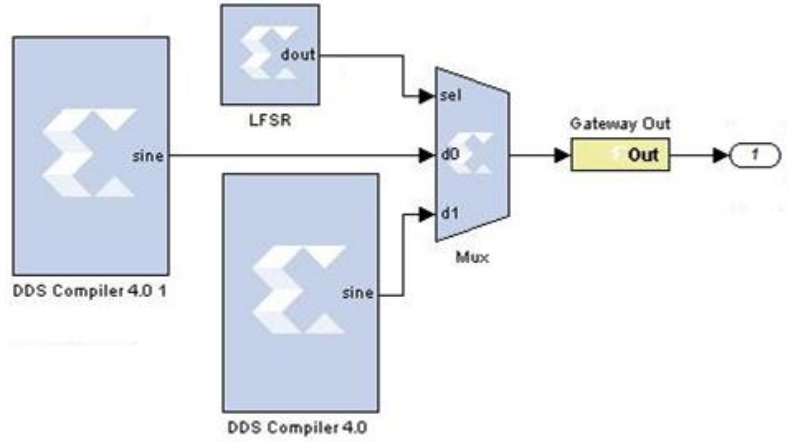

Figure 5. BPSK Modulator

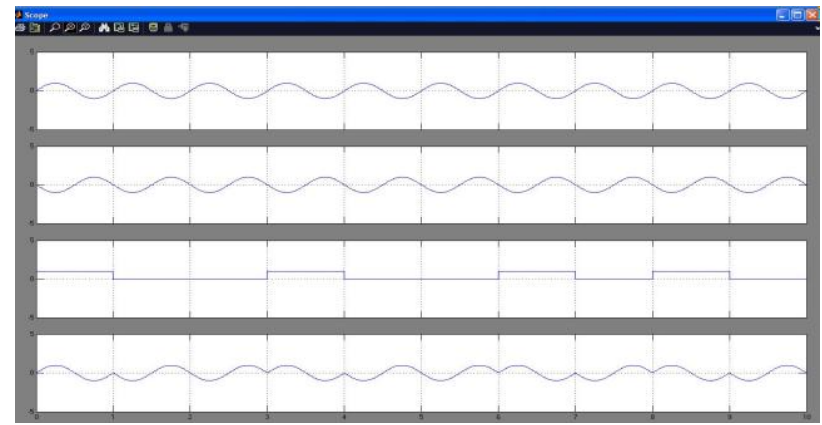

Figure 6.(1)carrier signal(2) $180^{\circ}$ phase shift carrier(3) modulating signal(4) modulated signal

\section{Design of BPSK Demodulator}

Block diagram of BPSK demodulator is shown in figure 7.The modulated signal which was transmitted by modulator is then pass through the channel and received at the input of demodulator. The carrier is recovered due to the DDS compiler and then multiplied with the modulated signal affected by noise. The obtained signal is then added with all the samples, multiplied, from a period. This operation takes place in the accumulator. Once we have a result, it is compared with a threshold.

If the compared signal is positive, the demodulator takes the decision that ' 1 ' was transmitted, otherwise, ' 0 ' is the result. Figure 8 illustrates the expected modulating signal generated in the modulator and the demodulated signal obtained after the demodulation operation.

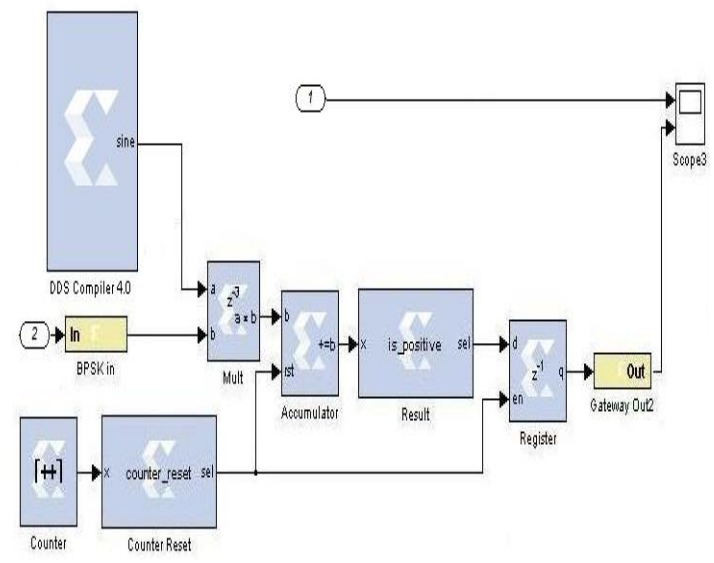

Figure 7. BPSK Demodulator 


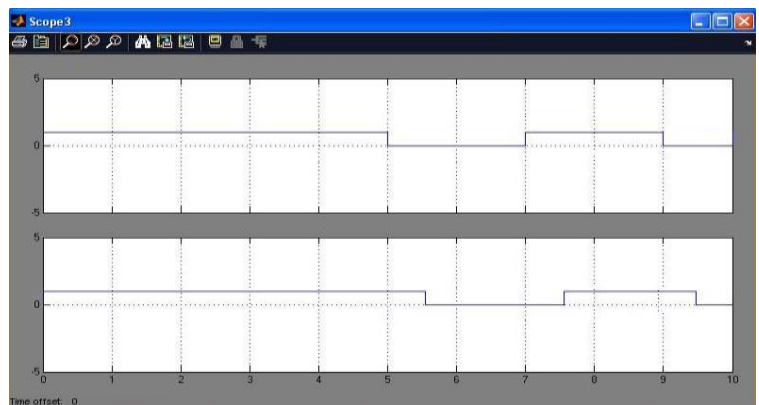

Figure 8. (a) The modulating signal (b) The demodulated signal

\section{Design of BPSK System On The SPARTAN 3E Board}

The BPSK system on FPGA kit is shown in figure 9. It consists of two Spartan 3E boards, first behaves as a modulator and the second one, as a demodulator. The connections between the two boards are made of three wires: first comports as a communication channel, the second as an asynchronous reset signal and the last one for the synchronization of the two boards.

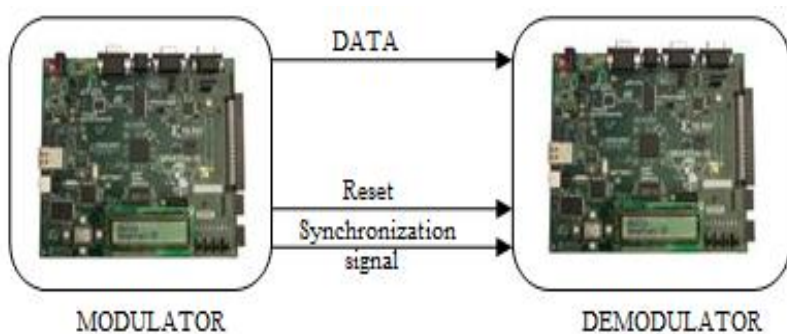

Figure 9 .BPSK System.

\section{1)Modulator part:}

\section{Results}

Fig 10(a) represent the linear feedback shift register which is use to generate the input data.fig 10(b) illustrate the output waveform and 8bit sequence of LFSR.

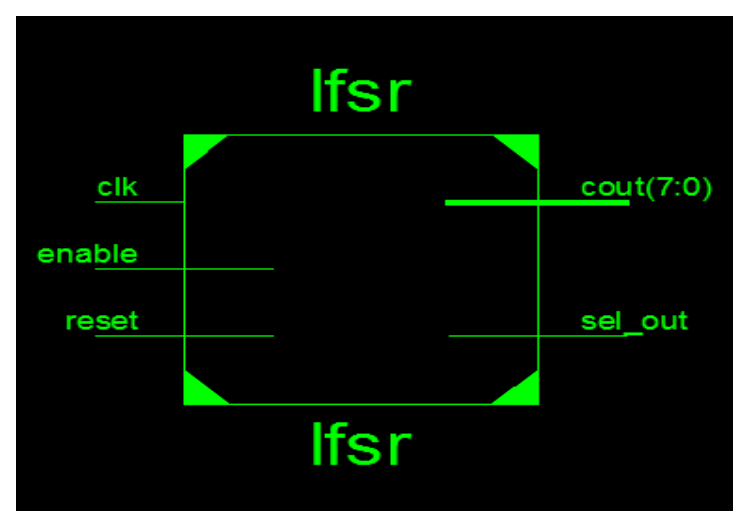

Fig 10 (a). LFSR RTL view

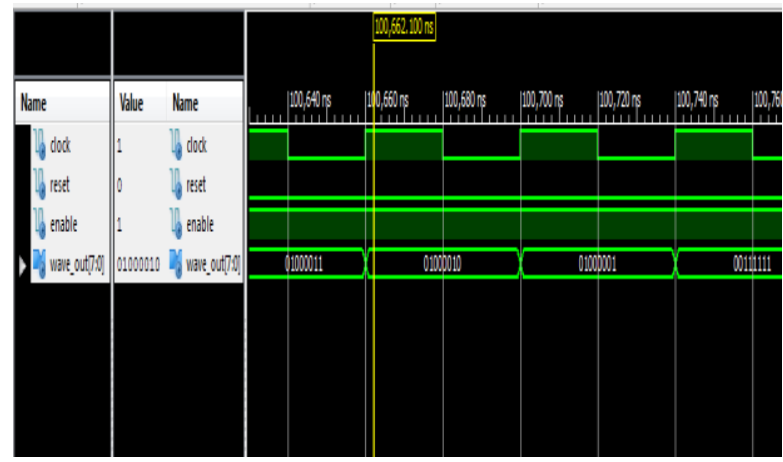

Fig 10 (b). LFSR Output waveform 
Fig 10(c) represent the carrier generation block which is use to generate the sinusoidal waveform..fig 10 (d) illustrate the sinusoidal waveform.

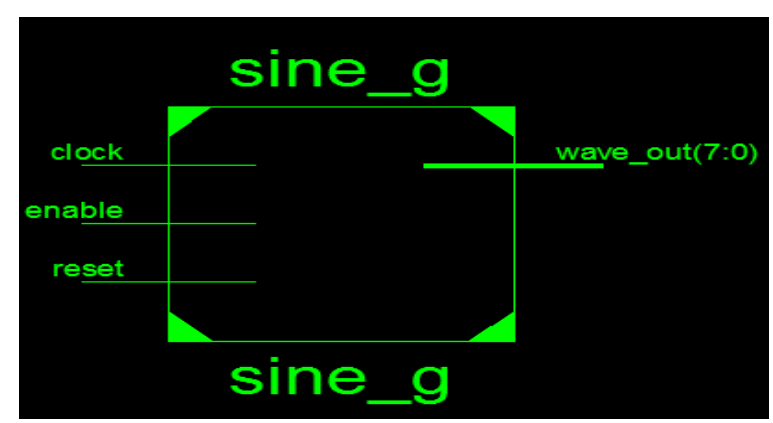

Fig 10 (c). RTL view of DDS Compiler

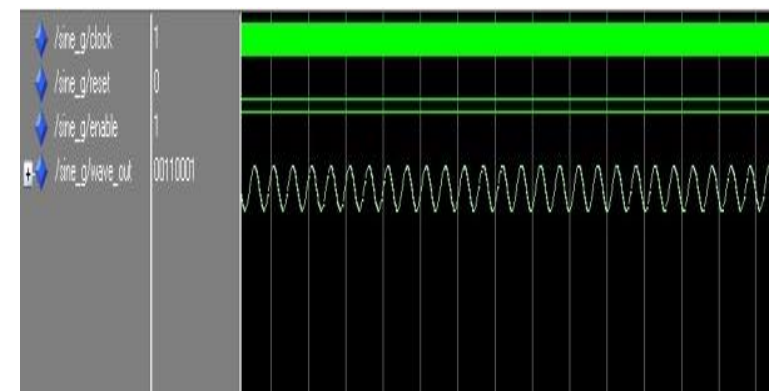

Fig 10 (d). DDS Compiler output waveform

Fig 10(e) shows the complete modulator block. The output waveform of modulator in Xilinx is shown in fig 10 (f).Fig 10(g) shows the modulator output using

Modelsim software to clearly see the sinusoidal waveforms.

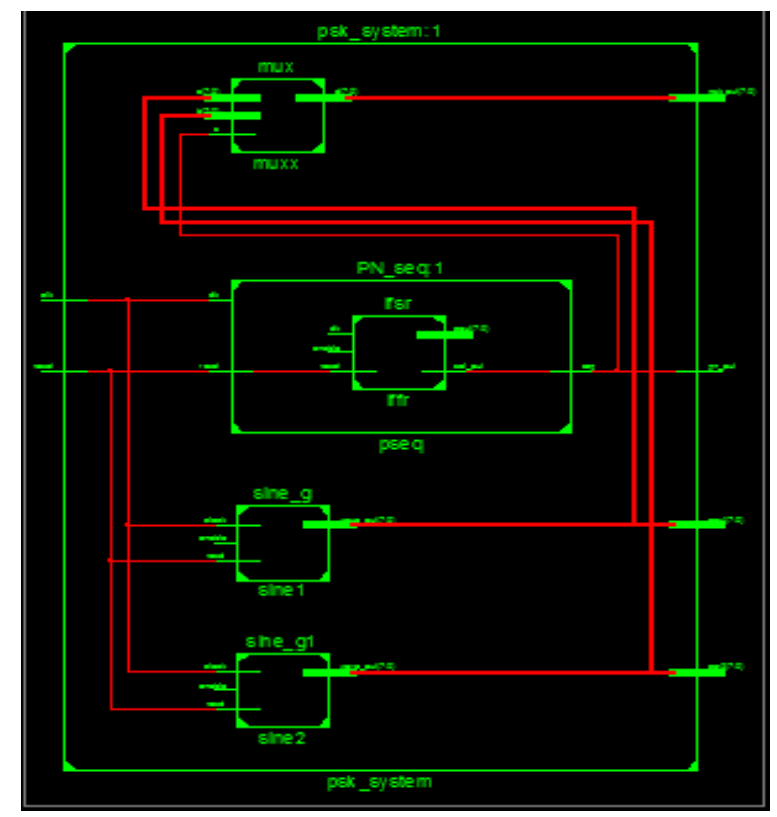

Fig 10 (e). RTL View of Modulator 


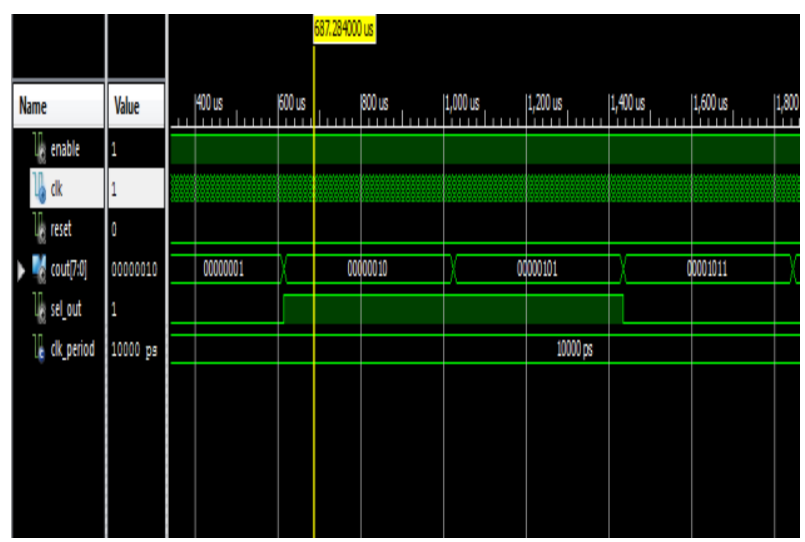

Fig $10(\mathbf{e})$. Modulator output in Xilinx software

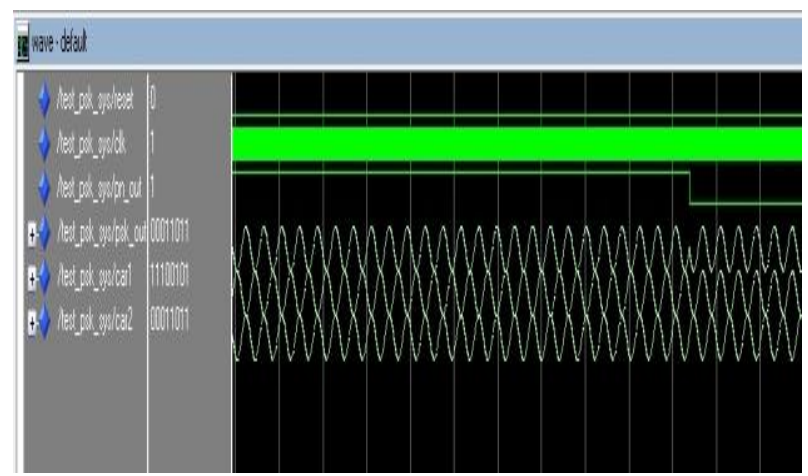

Fig 10 (g). Modulator output in modelsim software

\section{2)Demodulator part:}

Fig 11. Shows the complete demodulator block in which fistt block is the sine wave generation block which is use to recover the strength of input signal,second block is the multiplier and third block is the accumulator.

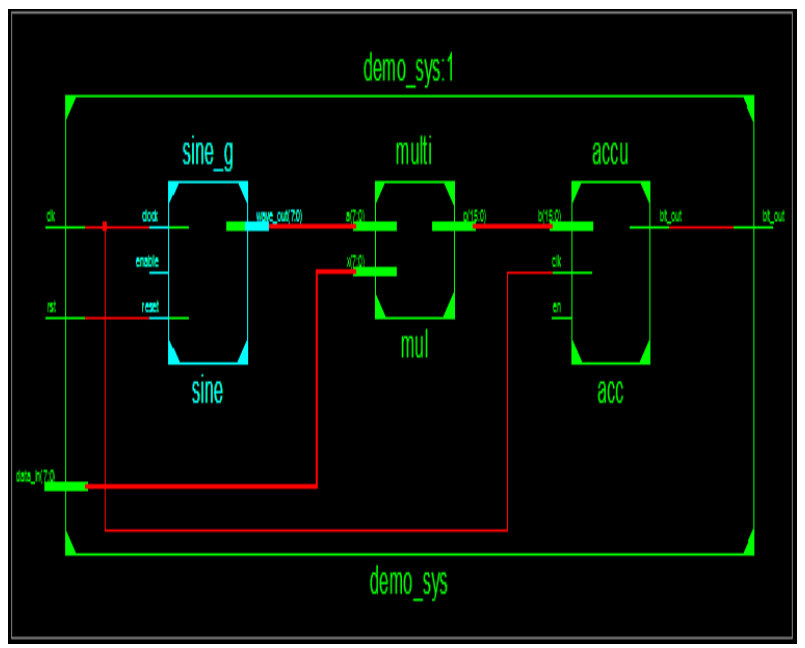

Fig 11.RTL View of Demodulator

\section{3) Combine modulator and demodulator System:}

Fig 12(a) represent the connection between modulator and demodulator. The output of modulator is given to the input of demodulator.Similarly fig 12(b) shows the internal blocks of modulator and demodulator.The final output of BPSK system is shown in fig 12(c) in which first signal is the input signal and second signal is the output of demodulator. 


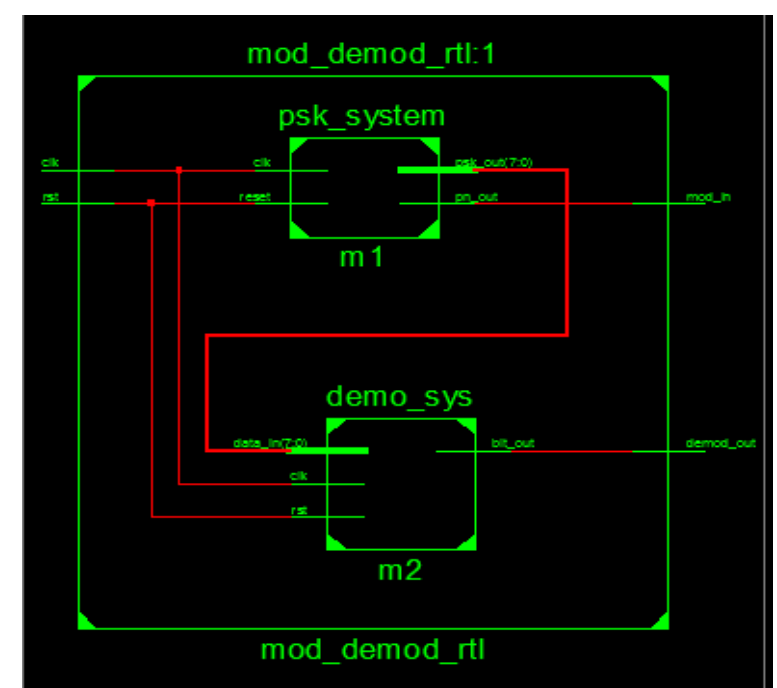

Fig 12 (a).RTL view of combine modulator and demodulator

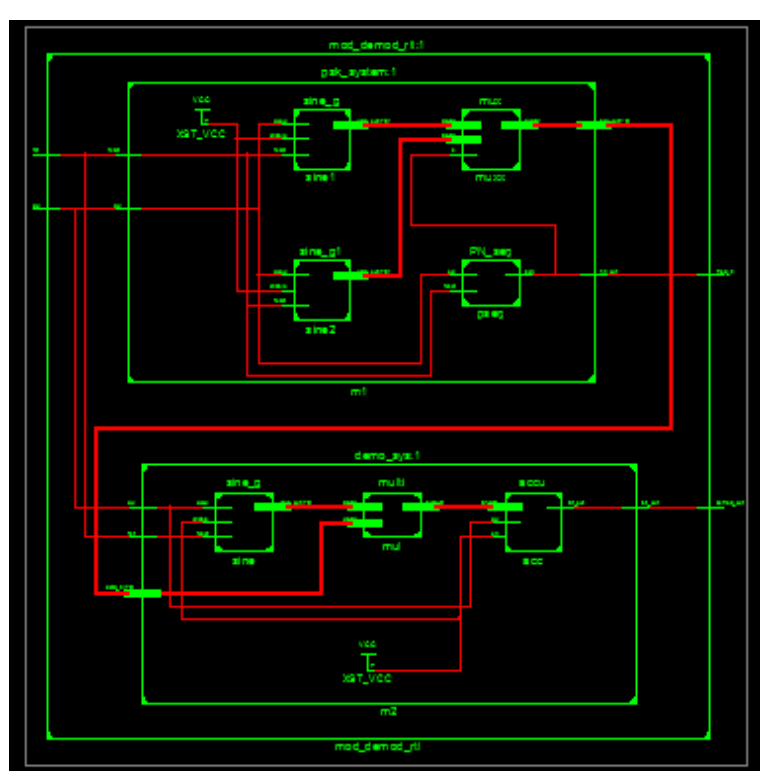

Fig 12(b). RTL View of modulator \& demodulator system

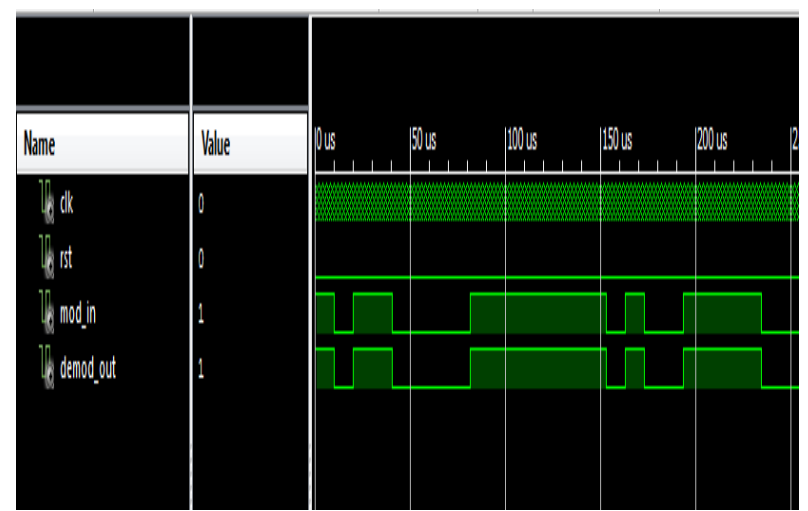

Fig 12 (c) Output waveform of modulator and demodulator

Fig.13 and 14 illustrate the design summary of modulator and demodulator board.The design summary shows the various synthesizer option that were enabled and some device utilization and timing statistics for the synthesized design. 


\begin{tabular}{|c|c|c|c|}
\hline \multicolumn{4}{|c|}{ Device Utilization Summary } \\
\hline Logic Utilization & Used & $\begin{array}{l}\text { Availabl } \\
\mathrm{e}\end{array}$ & $\begin{array}{l}\text { Utilizati } \\
\text { on }\end{array}$ \\
\hline Number of Slices: & 111 & 768 & $14 \%$ \\
\hline Number of Slice Flip Flops & 29 & 1536 & $1 \%$ \\
\hline Number of 4 input LUTs & 208 & 1536 & $13 \%$ \\
\hline Number used as logic & 207 & & \\
\hline Number used as Shift registers & 1 & & \\
\hline Number of IOs & 27 & & \\
\hline Number of bonded IOBs & 27 & 124 & $21 \%$ \\
\hline Number of GCLKs & 1 & 8 & $12 \%$ \\
\hline
\end{tabular}

Fig 13.Design Summary of the BPSK Modulator

\begin{tabular}{|l|l|l|l|}
\hline \multicolumn{4}{|c|}{ Device Utilization Summary } \\
\hline Logic Utilization & Used & $\begin{array}{l}\text { Availabl } \\
\mathrm{e}\end{array}$ & $\begin{array}{l}\text { Utilizati } \\
\text { on }\end{array}$ \\
\hline Number of Slices: & 157 & 768 & $20 \%$ \\
\hline Number of Slice Flip Flops & 51 & 1536 & $3 \%$ \\
\hline Number of 4 input LUTs & 305 & 1536 & $19 \%$ \\
\hline Number of IOs & 11 & & \\
\hline Number of bonded IOBs & 11 & 124 & $8 \%$ \\
\hline Number of GCLKs & 1 & 8 & $12 \%$ \\
\hline
\end{tabular}

Fig 14.Design Summary of the BPSK Demodulator

\section{Conclusion}

We represent the BPSK system (modulator and demodulator) using VHDL and implementation of the BPSK System on FPGA kit. The FPGA was selected as, compared to microcontrollers, it provides a larger number of input/output ports and the parallel implementation of hardware results in faster algorithm execution.

Both, the modulating signal and the carrier are generated internal, the modulating signal by a LFSR and the carrier by a DDS Compiler. The modulated signal is obtained at the output of a mux block and, then, passed through a communication channel where noise is added. In the demodulator, the carrier is recovered due to another DDS compiler and then multiplied with the modulated signal affected by noise. The obtained signal is then added with all the multiplied samples from the carrier in a period. The operation takes place in the accumulator. Once we have a result, it is compared with a decision threshold. If the compared signal is positive, the demodulator take the decision that ' 1 ' was transmitted, otherwise, ' 0 '.

\section{References}

[1]. S.O. Popescu, A.S.Gontean and G.Budura, "BPSK System on Spartan 3E FPGA" SAMI 2012 • 10th IEEE Jubilee International Symposium on Applied Machine Intelligence and Informatics • January 26-28, $2012 \cdot$ Herl'any, Slovakia.

[2]. F.Ahamed, A.Scorpino, "An educational digital communications project using FPGAs to implement a BPSK Detector", IEEE Transactions on Education, Vol.48, No.1, 2005, pp.191-197.

[3]. Thotamsetty M Prasad1 , Syed Jahangir" Hardware/Software Co-Simulation of BPSK Modulator Using Xilinx System Generator" IOSR Journal of Engineering (IOSRJEN) e-ISSN: 2250-3021, p-ISSN: 2278-8719, www.iosrjen.org Volume 2, Issue 10 (October 2012), PP 54-58

[4]. O.Azarmanesh, S.Bilen, "Developing a rapid prototyping method using a Matlab/ Simulink/ FPGA development to enable importing legacy code", Proceedings of the SDR "08 Technical Conference and product Exposition, USA, 2008.

[5]. Y.H.Chye, M.F.Ain, N.M.Zawawi, "Design of BPSK Transmitter Using FPGA with DAC", in Proceedings of the 2009 IEEE 9th Malaysia Conference on Communications, Malaysia, 2009, pp.451-456.

[6]. P.Dondon, J.M.Micouleau, J.Legall, .K.Kadionik, "Design of a low cost BPSK modulator/demodulator for a practical teaching of digital modulation techniques", in the 4th WSEAS/IASME International Conference on Engineering Education, Greece, 2007, pp.61-66.

[7]. P.Krivić, G.Štimac, FPGA Implementation of BPSK Modem for Telemetry Systems Operating in Noisy Environments, Proceedings of the 33rd International Convention on Information and Communication Technology, Electronics and Microelectronics, Croatia, 2010, pp.1727-1731. 